\section{Impact of the COVID-19 infection on the "benign" nature of the illness of Schönlein-Henoch}

\author{
A Vasileva1*, IV Chakarov² and P Chakarova1 \\ 'Trakia University, Medical Faculty, Clinic of Paediatrics, UMHAT "Prof. Dr. Stoyan Kirkovich", \\ Stara Zagora, Bulgaria \\ ${ }^{2}$ Trakia University, Medical Faculty, Clinic of Paediatrics, Clinic of Childhood Hematology and \\ Oncology, UMHAT "Tsaritsa Yoanna-ISUL”, Sofia, Bulgaria
}

\section{Abstract}

The incidence of COVID-19 in children has been variable. Although now the number of infected children worldwide, and in particular nationally, is small, they are not protected from the infection. Moreover, in the most severe cases septic shock, metabolic acidosis, irreversible bleeding, and coagulation dysfunction have been registered.

In February 2021 a 17-year-old boy showed for examination with complaints of pain in the upper part of the abdomen, accompanied by involvement of the ankle joints, the appearance of a rash on the lower extremities with the characteristic of hemorrhagic vasculitis, and a positive test for COVID-19. The characteristics of the skin purpura, the abdominal pain, and the arthralgia led us to the diagnosis of Schönlein-Henoch purpura.

The verification of past COVID-19 infection was done by the established high titer of specific IgG antibodies. The clinical evolution of the disease went beyond its generally accepted benign nature - the first manifestation of the illness had been followed by four more relapses, which necessitated new hospitalizations and a change in the therapeutic approach.

COVID-19 infection is the cause of a more aggressive course of vasculitis.

\section{More Information}

*Address for Correspondence: A Vasileva, Clinic of Paediatrics, UMHAT "Prof. Dr. Stoyan Kirkovich", Stara Zagora, Bulgaria,

Email: ani_asken@abv.bg

Submitted: December 23, 2021

Approved: January 10, 2022

Published: January 11, 2022

How to cite this article: Vasileva A

Chakarov IV, Chakarova P. Impact of the COVID-19 infection on the "benign" nature of the illness of Schönlein-Henoch. Int J Clin Virol. 2022; 6: 007-010.

DOI: 10.29328/journal.ijcv.1001042

Copyright: @ 2022 Vasileva A, et al. This is an open access article distributed under the Creative Commons Attribution License, which permits unrestricted use, distribution, and reproduction in any medium, provided the original work is properly cited.

Keywords: COVID-19; Henoch-Schönlein purpura; Abdominal pain

W) Check for updates

OPEN ACCESS

\section{Introduction}

Coronaviruses are a large family of viruses and a subset of Coronaviridae belonging to the genus Nidovirales, kingdom Riboviria, which range from the common cold virus to more severe cases of the disease, such as SARS, MERS, and COVID-19.

The incidence of COVID-19 in children has always been and will be very different from that in adults. Although the number of infected children worldwide, and in particular nationally, is currently small, they are not immune to the infection. In most cases, children with COVID-19 are asymptomatic or have mild symptoms without fever and pneumonia. The most severe cases may present with septic shock, metabolic acidosis, coagulation dysfunction, and irreversible bleeding.

A typical example of a severe course and unpredictable complications is the association of COVID-19 infection with Schönlein-Henoch vasculitis. Morbus Schoenlein has been known to be the most common acute systemic vasculitis in childhood, affecting mainly the skin, gastrointestinal tract, joints, and kidneys. The main clinical manifestation of
Schönlein-Henoch is purpura without thrombocytopenia, characterized by bilateral symmetrical distribution in both lower extremities, abdominal pain with/without blood in the stool, arthralgia, hematuria, and/or proteinuria $[1,2]$. The increased production of polymeric IgA by the mucosal immune system in response to mucosal antigens, such as bacteria, viruses, or fungi, is considered to be the main trigger. In the acute phase of the disease, concentrations of proinflammatory cytokines are elevated, which can cause endothelial damage [3]. The first excellent description of the disease was made by Heberden in 1801, and then by Villain in 1808.

Many years of wandering by many authors around the world have led them to the conclusion that SchönleinHenoch disease is an allergic disease and that there is nothing complicated about the pathogenesis, and that bleeding and clotting times are always normal. However, the most severe surgical complications of Schönlein-Henoch disease are hemorrhagic infarction and perforation of the small intestinal wall; as well as intramural hematoma and intestinal intussusception. Routinely used drugs are most 
often corticosteroids, but in the most severe cases, especially when the urinary system is involved, immunosuppressants and plasmapheresis are indicated.

The diagnosis of Schönlein-Henoch purpura is usually based on clinical features: skin rash, colic-like abdominal pain, arthralgia, and hematuria. In $10 \%$ to $40 \%$ of cases, gastrointestinal or joint symptoms may precede the appearance of purpura on the skin for up to 2 weeks, which is a mandatory diagnostic criterion [4]. Therefore, it may be difficult to diagnose Schönlein-Henoch disease when the patient shows atypical clinical manifestations. The interval between the onset of symptoms and the diagnosis can be between 1 and 30 days [5].

Although the prognosis of Schönlein-Henoch purpura is generally good, gastrointestinal involvement is a very serious potential complication, especially in cases of massive bleeding, intestinal infarction, perforation, intussusception, peritonitis. Surgical interventions related to gastrointestinal complications are performed in $5 \%$ to $12 \%$ of patients with the disease, and in recurrences, this percentage is reported to reach 30 [6].

This is not the case if the disease is associated with COVID-19 infection. In the clinical case presented by us, the evolution of the clinical course gives suspicion of a change in the concept of benign hemorrhagic illness.

\section{Materials and methods}

It is about a 17-year-old boy, who fell ill at the beginning of December 2020 with a runny nose, cough, and sore throat, having contact with COVID-19. A few days after the onset of these symptoms, swelling, and pain of the ankle joints appeared, hemorrhagic rash on the lower extremities, anterior abdominal wall. From the conducted laboratory tests-signs of moderate inflammatory activity (CRP $25 \mathrm{~g} / \mathrm{l}$ (normal ranges 0-6 g/l), WBC $17 \times 10^{9} / \mathrm{l}$, AST < 200 IU(< 200 IU), IgA 4,75 g/l (normal ranges: 0.4 - $4.5 \mathrm{~g} / \mathrm{l}$ ) C4 0, $28 \mathrm{~g} / \mathrm{l}$ (normal ranges: 0.1 - $0.4 \mathrm{~g} / \mathrm{l}$ ), RF 4,7 IU/ml (normal ranges: < $14 \mathrm{IU} / \mathrm{ml}$ ), Fib 4,3 g/l (2.0 - $4.0 \mathrm{~g} / \mathrm{l}), \mathrm{D}-$ dimer $0,29 \mathrm{mcg} / \mathrm{ml}$ (0.0-0.05 mcg/ml), Schönlein-Henoch disease was accepted as a diagnosis.

Despite the conducted symptomatic therapy, three relapses followed - with the manifestation of cutaneousabdominal form, with intense abdominal pain, unresponsive to antispasmodics. High titers of IgG-specific antibodies to COVID-19 were detected.

Due to the tense clinical course and the potential risk of severe gastrointestinal complications leading to the acute abdomen, levels of factor VIII (167\%) (Normal ranges: $60 \%$ - 150\%) and factor XIII (68\%) (Normal ranges: 70\% - 120\%) were also examined, with values outside the reference range. In this case, we can most likely assume that the COVID-19 virus was the cause of the high number of relapses, as no other etiological agent had been identified from the studies. Verification of past COVID-19 infection was based on the high titer of specific IgG antibodies found.

The recurrence of the abdominal manifestation of the disease and the changes found in the ultrasound (4.1-4.8 mm thick small intestinal wall and impaired differentiation) were an indication for initiating corticosteroid treatment (Figure 1).

Ultrasound follow-up was performed with HITACHI ARIETTA 70 ultrasound machine using a 5 to $7 \mathrm{MHz}$ highfrequency probe. Standard settings during the study included elevated levels of PRF and total gain. At the set basic settings during the examination, only normally vascularized intestinal mucosa without color or power Doppler signal is visualized.

After three weeks of corticosteroid administration and concomitant symptomatic therapy, factor XIII and VIII levels were within the reference range, ultrasound monitoring of changes in the small intestinal wall showed restoration of its thickness (2.4 mm) and restored differentiation (Figure 2).

For many years, the diagnostic and therapeutic approach in Schönlein-Henoch patients has been based on the assessment of clinical symptoms. The timing of initial corticosteroid treatment is also determined based on the severity of the abdominal pain. The duration comes down to stopping the abdominal pain syndrome. Variations in corticosteroid dosage usually correlate with the severity of abdominal pain, but a two-way relationship has been found between the inflammatory system and the coagulation system. The results of standard coagulation assays in Schonlein-Henoch, even in the acute phase, showed that aPTT and PT were within normal ranges. Anti-inflammatory cytokines and chemokines, as well as polymorphonuclear cells, platelets, fibrinogen, and fibrin, are important mediators in activating inflammation
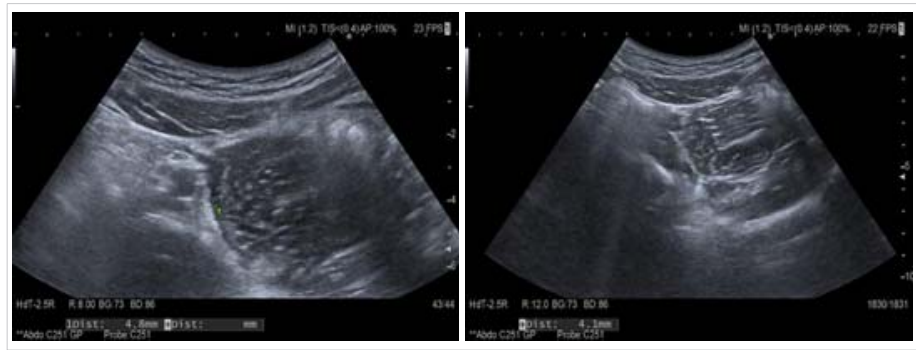

Figure 1: Abdominal ultrasound-small intestinal wall thickness.

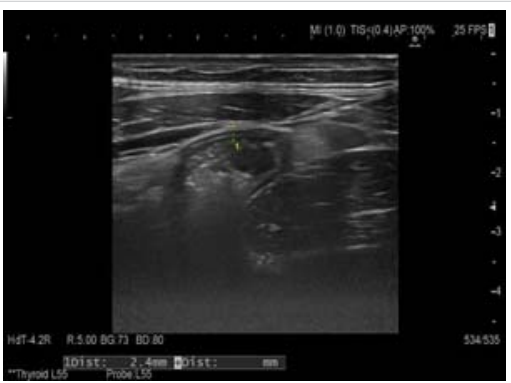

Figure 2: Follow-up abdominal ultrasound examination. 
and coagulation. Several studies have studied the involvement of these molecules in the pathogenesis of Schönlein-Henoch purpura [7-9]. To date, there are suggestions for their involvement in endothelial cell damage and subsequent activation of coagulation.

Factor XIII -fibrin-stabilizing factor decreases significantly during the acute phase of Schönlein-Henoch disease and can be used as a prognostic indicator $[10,11]$. The von Willebrand factor could be used as a specific marker for vascular damage and disease activity. The accumulated data on elevated von Willebrand factor concentrations are considered a reliable index of vascular damage and correlate with the concentration of circulating vWF: Ag [12]. The possible correlation of vWF: Ag with the size of the affected blood vessel has also been discussed [12-14]. The vWF: Ag has been shown not to play the role of an acute phase reagent but as a marker of vascular damage. Elevated vWF: Ag values reflect the increased amount of circulating endothelial factors due to vascular damage. Values return to normal when patients are in remission and remain above normal levels with continued symptoms. Von Willebrand factor assessment could be considered a reliable test for monitoring disease activity.

Ultrasound is effective as a first-line screening for children with gastrointestinal symptoms [15]. The normal intestinal wall consists of 5 layers, including the outermost echogenic mucosal and serosal layers, with submucosa, which manifests itself as a continuous homogeneous echogenic structure with a thickness of less than $2 \mathrm{~mm}$, delineated by two hypoechoic layers, inner-lamina propria, and outer-muscularis propria. Ultrasound sensitivity to detect intestinal abnormalities in children with Schönlein-Henoch purpura varies from $50 \%$ to $100 \%$ with undefined specificity [15-17]. It is an indisputable fact that intestinal sonography is the most accurate method for detecting intramural bleeding in this disease and can reduce diagnostic radiation in children. Findings most often demonstrate demarcated hypoechoic to echogenic crescentshaped areas, thickened intestinal walls, and the cockade phenomenon showing intramural bleeding and edema [17]. Ultrasonographic findings may help rule out the potential risk of unnecessary surgery, especially in cases where skin purpura occurs after gastrointestinal symptoms. Serial ultrasonography makes it possible to report the progressive reduction of thickening of the small intestinal wall, the recurrence of peristalsis, and visualization of intestinal folds [5].

\section{Discussion}

The need for a good knowledge of the aggression of the COVID-19 virus against organs other than the lung is the most likely cause of a large number of relapses in our clinical case, as well as serious endothelial damage to the intestinal wall. The possibility of monitoring the levels of f. VIII and f. XIII together with the ultrasound monitoring of the thickness of the small intestinal wall and the presence of intramural hemorrhages allows for a dynamic change in therapeutic behavior: the time of initiation of corticosteroid treatment and the duration and optimal dosage.

The precision in the use of corticosteroid therapy in Schönlein-Henoch purpura is also useful in preventing side effects, steroid dependence, and recurrence. The goal of using corticosteroids in the treatment of gastrointestinal form is to administer low-dose steroids initially, followed by intravenous immunoglobulin [18]. Perhaps the use of factor XIII for rapid improvement in severe abdominal pain and gastrointestinal bleeding would be good in the future $[19,20]$.

As the presented clinical case- with three relapses, due to the existing potential risk of involvement of the urinary system, the monitoring of the levels of factor XIII and vWF: Ag is extremely important.

Although Schönlein-Henoch's purpura has been considered to be a self-limiting disease, the patient we described, where there is also an association with COVID-19 infection, shows that it may not always be so.

\section{References}

1. Shi JS, Gu JY, Wang M. Clinical manifestations, gastroscopic, and pathological features of abdominal type Henoch-Schonlein purpura in children. Pediatr Nephrol. 2013; 28: 1424-1424.

2. Hung SP, Yang YH, Lin YT, Wang LC, Lee JH, et al. Clinical manifestations and outcomes of Henoch-Schönlein purpura: comparison between adults and children. Pediatr Neonatol. 2009; 50: 162-168. PubMed: https://pubmed.ncbi.nlm.nih.gov/19750891/

3. Mills JA, Michel BA, Bloch DA, Calabrese LH, Hunder GG, et al. The American College of Rheumatology 1990 criteria for the classification of Henoch-Schönlein purpura. Arthritis Rheum. 1990; 33: 1114-21. PubMed: https://pubmed.ncbi.nlm.nih.gov/2202310/

4. Davies PJ. Mozart's death: a rebuttal of Karhausen: further evidence for Schönlein-Henoch syndrome. J R Soc Med. 1991; 84: 737-740. PubMed: https://www.ncbi.nlm.nih.gov/pmc/articles/PMC1295524/

5. Couture A, Veyrac C, Baud C, Galifer RB, Armelin I. Evaluation of abdominal pain in Henoch-Schönlein syndrome by high frequency ultrasound. Pediatr Radiol. 1992; 22: 12-17.

PubMed: https://pubmed.ncbi.nlm.nih.gov/1594303/

6. Martinez-Frontanilla LA, Haase GM, Ernster JA, Bailey WC. Surgical complications in Henoch-Schönlein Purpura. J Pedaitr Surg. 1984; 19: 434-436.

PubMed: https://pubmed.ncbi.nlm.nih.gov/6481588/

7. BesbasN,SaatciU,RuacanS, OzenS,SungurA, etal. Theroleofcytokines in Henoch-Schonlein purpura. Scand J Rheumatol. 1997; 26: 456-460. PubMed: https://pubmed.ncbi.nlm.nih.gov/9433407/

8. Saulsbury FT. Henoch-Schönlein purpura. Curr Opin Rheumatol. 2010; 22: 598-602.

PubMed: https://pubmed.ncbi.nlm.nih.gov/20473173/

9. Del Vecchio GC, Penza R, Altomare M, Picante L, Aceto G, et al. Cytokine pattern and endothelium damage markers in Henoch-Schönlein purpura. Immunopharmacol Immunotoxicol. 2008; 30: 623-629. PubMed: https://pubmed.ncbi.nlm.nih.gov/18668398/

10. Dalens B, Travade P, Labbé A, Bezou MJ. Diagnostic and prognostic value of fibrin stabilising factor in Schönlein-Henoch syndrome. Arch Dis Child. 1983; 58: 12-14.

PubMed: https://pubmed.ncbi.nlm.nih.gov/6830268/ 
11. Kamitsuji H, Tani K, Yasui M, Taniguchi A, Taira K, et al. Activity of blood coagulation factor XIII as a prognostic indicator in patients with Henoch-Schönlein purpura. Efficacy of factor XIII substitution. Eur J Pediatr. 1987; 146: 519-523.

PubMed: https://pubmed.ncbi.nlm.nih.gov/3678279/

12. Bowyer SL, Ragsdale CG, Sullivan DB. Factor VIII related antigen and childhood rheumatic disease. J Rheumatol. 1989; 16: 1093-1097. PubMed: https://pubmed.ncbi.nlm.nih.gov/2585406/

13. Nusinow SR, Federici AB, Zimmerman TS, Curd JG. Increased von Willebrand factor antigen in the plasma of patients with vasculitis. Arthritis Rheum. 1984; 27: 1405-1410.

PubMed: https://pubmed.ncbi.nlm.nih.gov/6508862/

14. Woolf AD, Wakerley G, Wallington TB, Scott DGI, Dieppe PA. Factor VIII related antigen in the assessment of vasculitis. Ann Rheum Dis. 1987; 46: 441-447.

PubMed: https://pubmed.ncbi.nlm.nih.gov/3115202/

15. Vasavada P. Ultrasound evaluation of acute abdominal emergencies in infants and children. Radiol Clin North Am. 2004; 42: 445-456. PubMed: https://pubmed.ncbi.nlm.nih.gov/15136027/

16. Ozdemir $\mathrm{H}$, Isik $\mathrm{S}$, Buyan N. Sonographic demonstration of intestinal involvementinHenoch-Schönleinsyndrome. EurJRadiol. 1995;20:32-34. PubMed: https://pubmed.ncbi.nlm.nih.gov/7556249/

17. Bomelburg $\mathrm{T}$, Claasen $\mathrm{U}$, von Langerke HJ. Intestinal ultrasonographic findingsin Schönlein-Henochsyndrome. EurPediatr. 1991;150:158-160. PubMed: https://pubmed.ncbi.nlm.nih.gov/2044583/

18. Yang HR, Choi WJ, Ko JS, Seo JK. Intravenous immunoglobulin for severe gastrointestinal manifestation of Henoch-Schönlein purpura refractory to corticosteroid therapy. Korean Pediatr. 2006; 69: 798-806. PubMed: https://pubmed.ncbi.nlm.nih.gov/27133370/

19. Ebert EC. Gastrointestinal manifestations of Henoch-Schonlein purpura. Dig Dis Sci. 2008; 53: 2011-2019.

PubMed: https://pubmed.ncbi.nlm.nih.gov/18351468/

20. Fukui $H$, Kamitsuji $H$, Nagao T, Yamada K, Akatsuka J, et al. Clinical evaluation of a pasteurizes factor XIII concentrate administration in Henoch-Schönlein purpura. Thromb Res 1989; 56: 447-75. 